\title{
Maxillofacial Radiology 185
}

SADJ October 2020, Vol. 75 No. 9 p511

CJ Nortjé

Below are cropped radiographic images of various lesions that may present in the jaws of a patient during a radiographic examination. What is the cardinal feature that are present in every case presented?
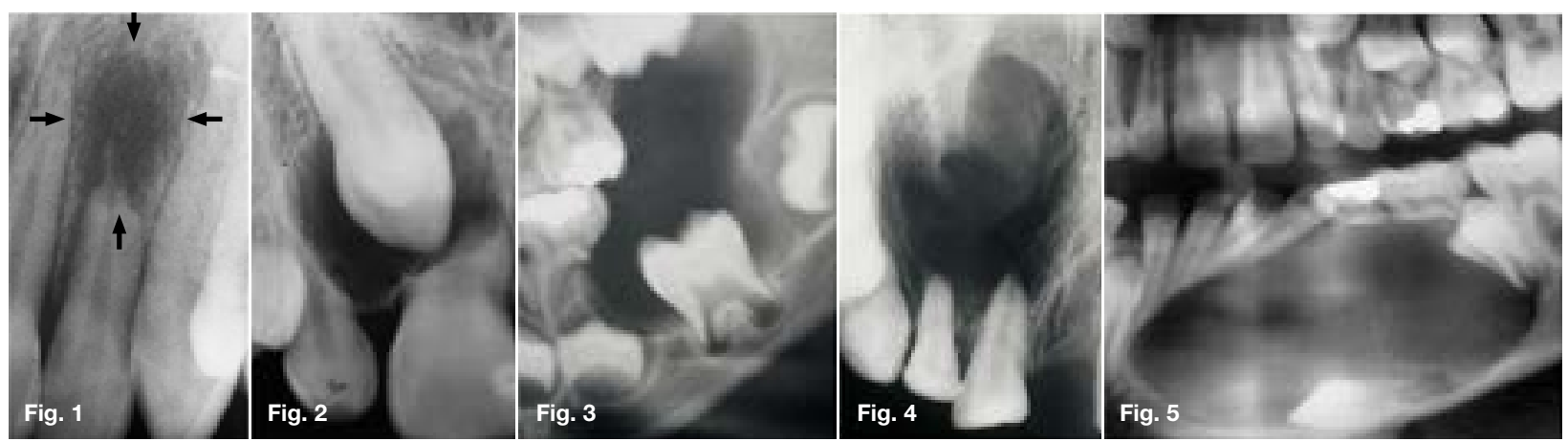
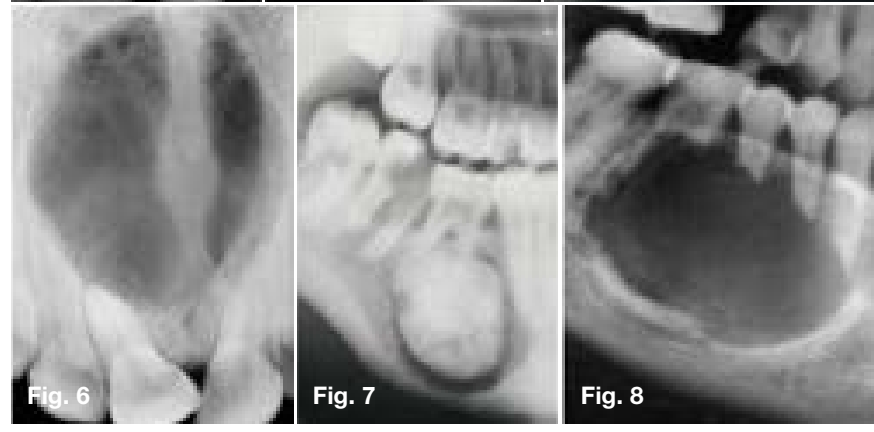
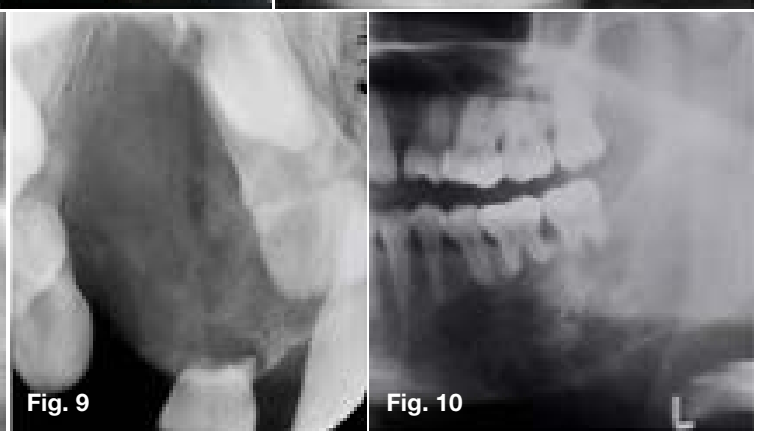

INTERPRETATION

The cardinal feature that is discernible in every case is the presence of external root resorption. Resorption of teeth occurs in many circumstances other than the normal process associated with the shedding of deciduous teeth. Since resorption of a tooth may begin either on the external surface or on the inside of the tooth, general terms such as "external resorption" and "internal resorption" are used to distinguish between the two types. The purpose of this communication is to discuss external resorption in the presence of certain lesions. Resorption of calcified dental tissue occurs in the same fashion as that of bone and, in most instances, the presence of osteoclasts is an outstanding feature in areas of active resorption, however there is considerable evidence that osteoclasts may not be essential for the resorption. Periapical granuloma resulting from death of pulp caused by traumatic injury resulted in resorption of the roots (Fig. 1). Resorption also occurs as a result of pressure exerted by an impacted tooth (Fig. 2). Roots of primary teeth may undergo resorption due to the eruption force of an eruption cyst (Fig. 3). An apical cyst arising as a result of pulp infection may exert pressure on the apex of the involved or adjacent tooth that the intervening connection tissue is stimulated, osteoclasts form and resorption begins (Fig. 4). Benign and malignant tumours may cause root resorption. Although a benign lesion such as a dentigerous cyst

Christoffel J Nortjé: $B C h D, P h D, A B O M R, D S c$. Faculty of Dentistry, University of the Western Cape.

ORCID Number: 0000-0002-9717-5514

Email: cnortje@uwc.ac.za
(Fig. 5) are more likely to produce displacement than destruction of the tooth. In most cases connective tissue present between the tumour and the tooth and it is from this tissue that the osteoclasts develop which appear responsible for the root resorption. Cysts cause root resorption in a manner similar as benign tumours chiefly by pressure as demonstrated by the nasopalatine duct cyst (Fig. 6) causing resorption of the central incisors. Figure 7 shows a cementoblastoma causing resorption of the mesial root of the first molar tooth. A classic example of the knife edge resorption normally seen in an amelobalstoma is demonstrated in Figure 8. Extensive root resorption is demonstrated by a central giant cell tumour (Fig. 9). Malignant tumours may also cause root resorption. Figure 10 is a longstanding chondrosarcoma and resorption showing a mixed radiolucent/opaque lesion with rounded, speckled, mottled or flocculent calcifications, periosteal new bone perpendicular to original cortex and resorption of the roots of the 36 and 37 . Resorption is rarely important but very occasionally results from some destructive disease such as a tumour. Pressure is probably the main factor. However, in some cases no cause is apparent. Resorption is mainly carried out by osteoclasts which, during active phases, may be seen in the lacunae in the hard tissue with which their cytoplasm to merge. When resorption is very slow, osteoclasts may be seen and, being intermittent in their action, disappear during the inactive periods.

\section{Reference}

1. Shafer, WG, Hine, MK, Levy, BM. A Textbook of Oral Pathology, $3^{\text {rd }}$ ed. W.B. Saunders Company, Philadelphia \& London. 1974: 295-99. 